\title{
Visualization of Quantum Dots by Freeze-fracture Electron Microscopy
}

\author{
B. Papahadjopoulos-Sternberg*, K. C. Weng**, and J. W. Park** \\ *NanoAnalytical Laboratory, 3951 Sacramento Street, San Francisco, CA 94118, USA \\ **Hematology/Oncology, UCSF Cancer Center, 2340 Sutter Street, San Francisco, CA 94115, USA
}

Semiconductor quantum dots (QDs) are a new class of promising fluorescent probes for many biological and biomedical applications due to their advantages over conventional dyes [1]. They exhibit excellent photostability and their emission spectra are narrow, symmetrical, and tunable according to their size and material composition, allowing closer spacing of different probes without spectral overlap. Independently, tremendous effort has been dedicated to design and optimize nanoscale targeted drug delivery systems (tDDS) including target-specific liposomal therapeutics displaying remarkable anti-tumor efficacy and relatively mild toxicity profiles [2,3]. Our aim is to combine both fruitful developments by incorporation of QDs into immunoliposome nanocomplexes to develop novel molecular imaging agents for applications in cancer diagnosis and treatment.

Freeze-fracture electron microscopy (ff-em) is a powerful technique to characterize nanometer- and micrometer-size drug/gene carriers including tDDS [4-10]. Using ff-em we visualized carboxyl QDs, naked and complexed with liposomes as well as with HER-2-tageting Ils.

As visible on Fig. 1 A and B naked QD appear as small, mostly spherical particles on freeze-fracture electron micrographs. The diameters of the QDs investigated (Q21301MP from Invitrogen Corp.) are ranging from 5 to $22 \mathrm{~nm}$ with a high population around $11 \mathrm{~nm}$. As visible on all freeze-fracture electron micrographs (Fig. 1 A,B and 2 A,B) QD's always display their shadows behind the structure what is characteristic for hard-core particles. Fig. 2 shows QDs complexed with non-targeted liposomes (A) and HER-2-targeting Ils (B). The liposomes of both types display clearly convex and concave fracture planes (shadow behind and shadow in front of the structures respectively) what is characteristic for bilayer vesicles such as liposomes $[4,5]$. While the diameters of the non-targeted liposomes are mostly below $100 \mathrm{~nm}$ (main diameter $88.4 \pm 2 \mathrm{~nm}$ ) the targeted liposomes show slightly larger diameters (main diameter 108.8 $\pm 2 \mathrm{~nm}$ ). Especially in the QD-IL-complex preparation the QDs seem to decorate the contact areas between the liposomes riveting the liposomes closely together.

\section{References}

[1] X. Michalet et al., Science 30 (2005) 538.

[2] J. W. Park et al., Clinical Cancer Research 8 (2002) 1172.

[3] V. P. Torchilin, Nature Reviews Drug Discovery 4 (2005)145.

[4] B. Sternberg, Liposome Technology, CRC Press I (1992) 363.

[5] B. Sternberg, Handbook Nonmedical Applications of Liposomes CRC Press (1996) 271.

[6] B. Sternberg, Medical Applications of Liposomes, Elsevier (1998) 395.

[7] B. Sternberg et al., Nature 378 (1995) 21.

[8] V. P. Torchilin et al. PNAS (2003) 100 (4) 1972.

[9] V. P. Torchilin et al. PNAS (2003) 100 (10) 603.

[10] P. C. Bell et al., accepted by Biochemistry (2006) 


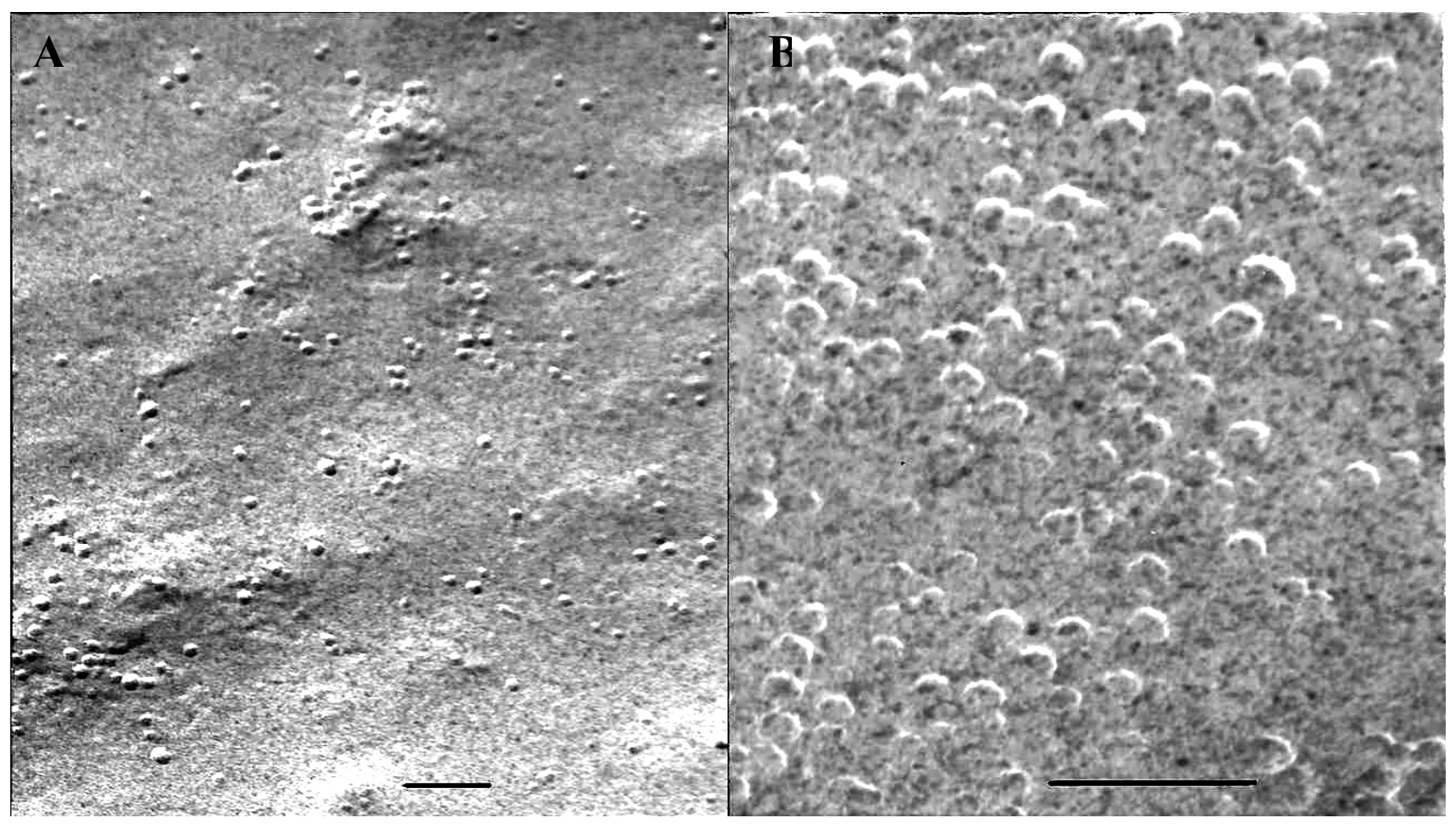

Fig. 1. Freeze-fracture electron micrograph of quantum dot controls. A. QDs at 19.300 x. B. QDs at $46.700 \mathrm{x}$. Scale bars $=100 \mathrm{~nm}$.

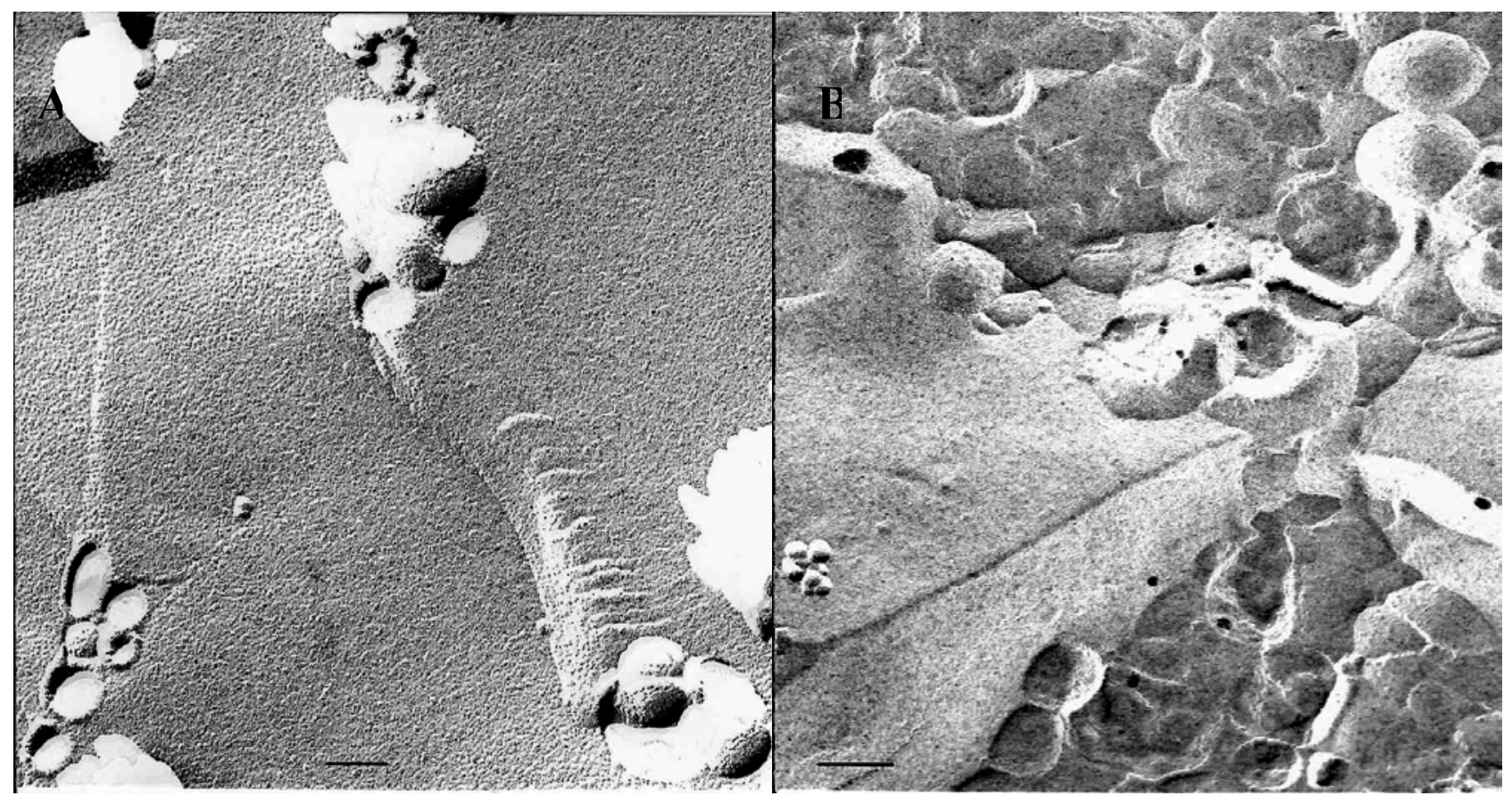

Fig. 2. Freeze-fracture electron micrographs of complexed quantum dots. A. Liposomal complexed QDs. B. QD-immunoliposome complexes (QD-Ils). Scale bars $=100 \mathrm{~nm}$. On all electron micrographs the shadow direction is running from bottom to top. 